\title{
The role of biological markers in predicting infertility associated with non-obstructive endometriosis
}

\author{
Nicolae Gica ${ }^{1,2} \mathbb{1}$, Anca Maria Panaitescu ${ }^{1,2}$, George lancu ${ }^{1,2}$,

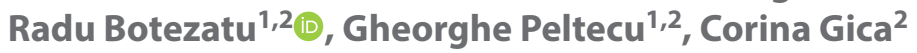 \\ 1"Carol Davila" University of Medicine and Pharmacy, Department of Obstetrics and Gynecology, Bucharest, Romania \\ ${ }^{2}$ Gynaecology Department — Filantropia Clinical Hospital, Bucharest, Romania
}

All authors had equal contributions to this article.

\begin{abstract}
Objectives: Our study evaluates if the use of biological markers can predict the infertility in women with non-obstructive endometriosis.

Material and methods:Two prospective, non-randomised studies were conducted to identify if CA-125, IL- 6 and IL- 8 can be used as predictive markers for infertility in women with non-obstructive endometriosis. Peripheral levels of CA-125, IL- 6 and IL-8 were measured before laparoscopy in all patients.

Results: We found a total number of 152 patients with non-obstructive endometriosis, we divided them in two groups: fertile and infertile women. There was a statistically significant difference of the mean of CA-125 values between the two groups $(p=0.00)$. The patients with infertility had a significantly higher IL-6 serum values than the fertile patients $(p=0.00)$. Regarding the IL-8 serum values, there was no statistically significant difference between the two groups fertile vs infertile, $(p=0.06)$. Conclusions: The elevated serum levels of CA-125 and IL- 6 was associated with an increased probability of being diagnosed with infertility. The IL-8 had no value in predicting infertility associated with non-obstructive endometriosis.
\end{abstract}

Key words: CA-125; IL-6; IL-8; biological marker; non-obstructive endometriosis; prediction of infertility

Ginekologia Polska 2020; 91, 4: 189-192

\section{INTRODUCTION}

Endometriosis affects $10 \%$ of women of reproductive age and is defined as the presence of endometrial tissue (stroma and glands) outside the uterine cavity [1, 2]. Very often is difficult to diagnose the endometriosis disease in the general population, due to non-specific symptoms [3]. Endometriosis negatively impairs the quality of life in women, through the presence of symptoms including dysmenorrhea, dyspareunia, chronic pelvic pain, dyschezia and dysuria [4] Non-invasive diagnosis tests were highly researched for endometriosis disease and many biological markers have been tested from peripheral blood as biomarkers of endometriosis. The patients with endometriosis associate infertility in 30 to $50 \%$ of cases, and about 25 to $50 \%$ of infertile women are diagnosed with endometriosis $[5,6]$.

The etiopathogenesis of endometriosis involves several theories. However, two conditions are common: a chronic pelvic and systemic inflammation stimulating the peritoneum secretion of cytokines and specifically Interleukin 6 (IL-6) and Interleukin 8 (IL-8) [7]. It has been suggested that the autoimmune mechanisms may play an important role in infertility [8].

So far, most studies are evaluating the biomarkers in the non-invasive diagnosis of endometriosis however, there are no correlation of this biomarkers with infertility in this population.

The aim of our study was to identify from peripheral blood frequent biomarkers correlated with endometriosis and infertility. CA 125 is the main biomarker along with proinflammatory cytokines detected in endometriosis. Among serum cytokines IL- 6 and IL-8 alone or in combination are known to be associate with endometriosis. Therefore, we hypothesized that this combination of IL- 6 and IL-8 with serum CA125 levels will predict infertility in women with endometriosis with a superior value than CA 125 alone $[7,8]$. 


\section{MATERIAL AND METHODS}

After receiving the approval of the Commission for Medical Ethics (Filantropia Hospital) we started two studies. In the first study CA-125 was tested in 116 women with non-obstructive endometriosis and in the second, IL-6 and IL- 8 were tested in 36 women. We collected plasma samples from women with non-obstructive endometriosis, prior to anesthesia induction, centrifuged at $4500 \mathrm{rpm}$ for 5 minutes at $4^{\circ} \mathrm{C}$, and stored at $-70^{\circ} \mathrm{C}$. The relevant information for each patient was collected in the electronic database. In each study patients were divided into two groups fertile and infertile women. The inclusion criteria were women with non-obstructive endometriosis, aged between 20 to 45 years old. A total number of 152 women have met the inclusion criteria. Included patients were women in which surgery was indicated for endometriosis, infertility work-up or ovarian mass and endometriosis was assessed by the surgeon based on the direct visualization of typical macroscopic endometriosis lesions. Infertile women were patients who require assisted conception after failure to achieve a clinical pregnancy within 12 months or more of regular unprotected sexual intercourse. The exclusion criteria from our studies were: women who have been operated within 3 months prior the sample collection, or with pelvic/systemic inflammatory disease. No confounders factors were included in the study.

We conducted a case-control experimental study, in which two independent groups of fertile and infertile patients were identified and were compared in terms of CA125, IL-6 and IL-8 markers (Fig. 1). Because it was an experimental study, there was no patient randomization process, the patients being assigned to the appropriate group based on the pathology or absence of the pathology.

A total of 116 patients with non-obstructive endometriosis were enrolled in the first study. All patients had non-obstructive endometriosis detected intraoperatively. Preoperatively, peripheral blood samples were collected and processed according to the laboratory protocol. The surgical approach was by laparoscopy in 113 (97.41\%) cases and by laparotomy in 3 (2.58\%) cases due to technical difficulties. The patients were classified according to their fertility as follows: 22 patients with unknown fertility (18.96\%), 54 preserved fertility patients $(46.55 \%), 36$ patients with primary infertility (31.03\%), 4 patients with secondary infertility (3.44\%).

After the exclusion of the patients with unknown fertility $(n=22)$, two groups were established, depending on the fertility status: fertile and infertile (with primary or secondary infertility).

The data were processed statistically using IBM SPSS 18. Quantitative variables were described in terms of central tendency, dispersion, and their distribution. According to their distribution the Mann-Whitney U test or the Student test was used for comparison between groups, while either Spearman's coefficient or Pearson's coefficient was used to characterize the correlations between variables. In this study there were no missing values.

\section{RESULTS}

Study 1. The role of CA-125 in predicting infertility associated with non-obstructive endometriosis

The mean age $( \pm S D)$ of fertile patients was 36.69 ( \pm 5.58 ), while the mean age $( \pm S D)$ of those with infertility was 36.43 ( \pm 4.56 ). In the group of fertile patients, the youngest age was 25 years and the oldest age was 45 years, similar values being also observed in the group of those diagnosed as infertile (respectively 26 years, 45 years). Regarding the age there were no statistically significant differences between the two groups $(p=0.81>0.05)$. The American Society for Reproductive Medicine (ASRM) scoring system was used in

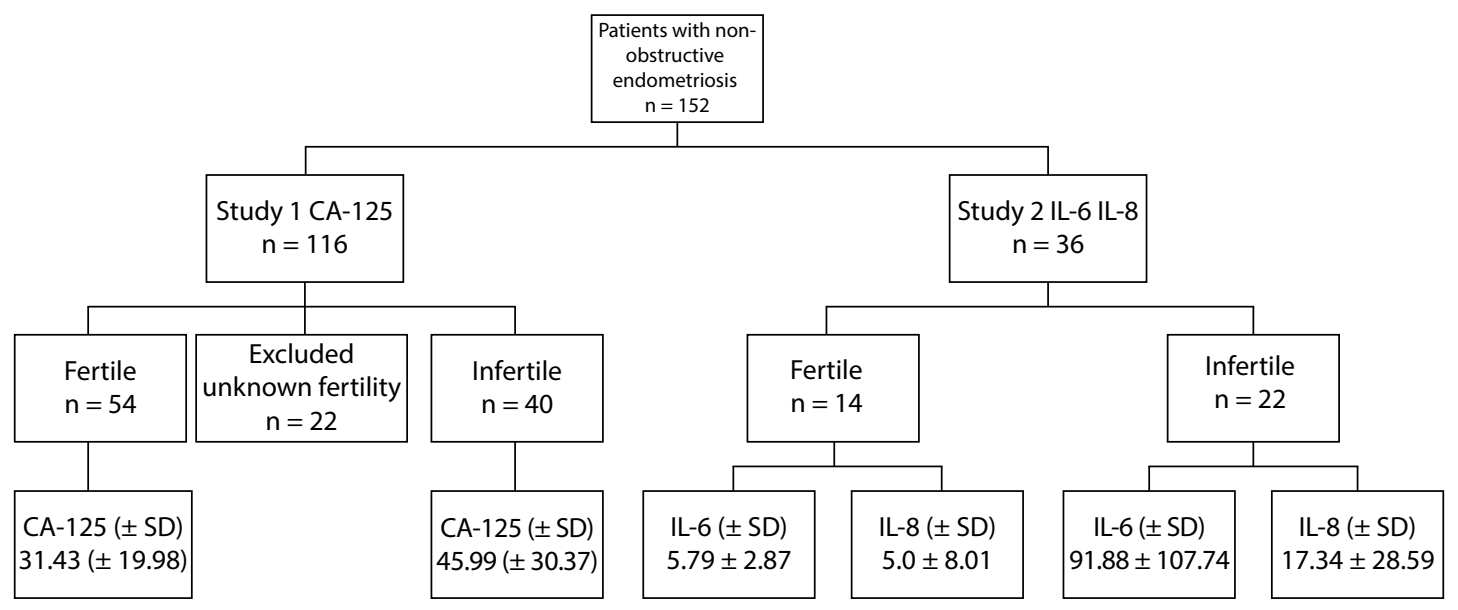

Figure 1. Flow Chart of the study design for the two studies 


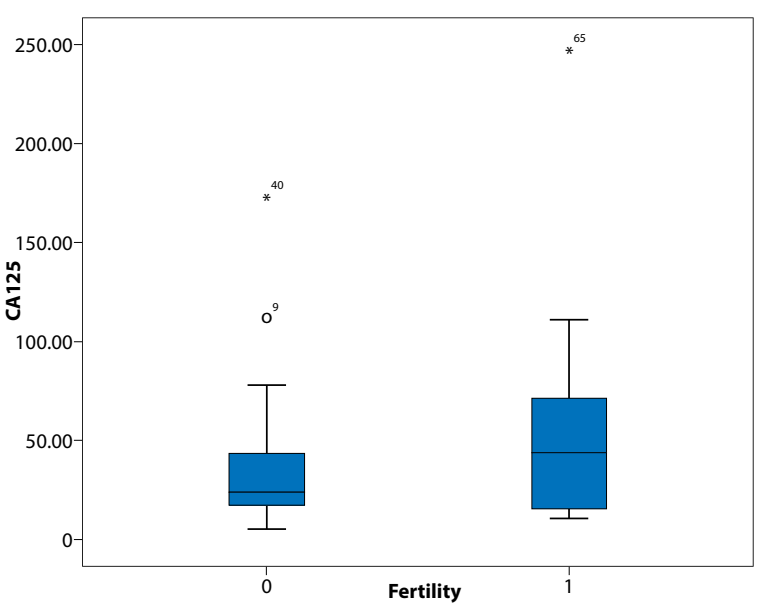

Figure 2. CA-125 values distribution in the two groups: fertile and infertile

order to stage endometriosis as follows: 15 patients (12.93\%) were enrolled in stage I, 57 patients $(49.13 \%)$ in the stage II, 30 patients $(25,86 \%)$ in stage III and 14 patients $(12.06 \%)$ in stage IV. There was no statistically significant influence of the stage of endometriosis in fertility ( $p=0.83>0.05)$. In the group of fertile patients, the mean values of CA-125 $( \pm$ SD) were 31.43 ( \pm 19.98 ) and median was 24 , while in the group of those diagnosed with infertility, the mean value ( \pm SD) was 45.99 ( \pm 30.37) and median 43.8 (Fig. 2). The distribution of CA- 125 values in the group of fertile patients was non-parametric $(p=0.00<0.05)$ and parametric for the infertile patients' group. There was a statistically significant difference of the mean values of the CA-125 between the two groups $(p=0.00)$.

\section{Study 2. The role of IL-6 and IL-8 in predicting infertility associated with non-obstructive endometriosis}

A total of 36 patients with non-obstructive endometriosis were identified as meeting our inclusion criteria. Laparoscopy was the surgical approach in all patients. Regarding their reproductive status, they were divided as follows: 22 (61.11\%) women with infertility and 14 (38.88\%) with normal fertility. Regarding the age of the enrolled women, the mean ( \pm SD) age of the patients without fertility problems was $32.43 \pm 7.73$, and of those diagnosed with infertility was $30.82 \pm 5.19$, the medians being equal, respectively 31 . In both groups, fertile and infertile, the distributions were non-parametric. In order to see if the stage of endometriosis influenced fertility status, a Chi-square test was conducted. This showed that in our group there was a statistically significant influence of the stage of endometriosis on fertility $(X 2=23.19), p=0.00$.

The mean values of IL- 6 ( \pm SD) in fertile patients were $5.79 \pm 2.87$ and the median was 6.01 and the infertility group the mean values was $91.88 \pm 107.74$ and the median was
100. The statistical analysis of the IL- 6 and IL- 8 reveals that the mean value of IL-6 ( \pm SD) was $38.38 \pm 87.86$ and the median of 10.5; Kolmogorov-Smirnov and Shapiro-Wilknormality tests reveals that the IL-6 values had a non-parametric distribution $(p=0.00)$.

For the IL 8 , the mean value ( \pm SD) was $12.54 \pm 23.58$ and the median of 1.6. Normality tests, Kolmogorov-Smirnov and Shapiro-Wilk, indicated a nonparametric distribution for IL-8 values. The correlation test (Spearman) demonstrates a statistically significant positive association $(p=0.00)$ between the two variables. The mean values of IL-8 ( \pm SD) in fertile patients were $5.00 \pm 8.01$ and the median was 1.1 and in the infertility group $17.34 \pm 28.59$ respectively, with a median of 3.53. The distribution of the IL-8 values was non-parametric in both groups. Women with infertility had significantly higher IL-6 median values than fertile patients $(p=0.00)$. There was no statistically significant difference between the median of IL-8 values in the two groups $(p=0.06)$. The prediction of a patient's probability of being diagnosed with infertility, requires a logistic regression analysis: the sensitivity was $75 \%$ and the specificity $90 \%$. The positive predictive value was $85.71 \%$ and the negative predictive value was $81.81 \%$.

In our studies the exposure was the CA-125, IL- 6 and IL- 8 testing. We calculated the following parameters: the sensitivity was $68.75 \%$, the specificity was $61.90 \%$, the positive predictive value was $84.61 \%$ and the negative predictive value was $39.39 \%$. After the statistical analysis we concluded that the CA-125 variable was the only one that contributed statistically significantly to this model $(p=0.01)$. The elevated values of this marker were associated with an increased probability of being diagnosed with infertility.

\section{DISCUSSION AND CONCLUSIONS}

Previous studies have demonstrated that the levels of cytokines and glycoproteins, are highly relevant for endometriosis, and an association of biomarkers represent non-invasive tests for endometriosis [9]. The CA-125 is a peripheral biomarker of endometriosis, produced by endometrial and mesothelial cells [10]. The IL-6 and IL-8 have elevated peripheral levels in women with endometriosis [11-14].

The aim of our studies was to evaluate the role of biomarkers in predicting infertility in patients with non-obstructive endometriosis. The peripheral blood biomarkers have been generally investigated for endometriosis, but not for non-obstructive endometriosis. Present data from the literature is controversial, with some studies demonstrating elevated interleukins, IL- 6 and IL 8 , in patients with endometriosis, while others have reported similar results in the endometriosis group versus control group $[3,4,11,13]$.

Through logistic regression, we tried to estimate the probability of a patient being infertile based of the CA-125 values. The CA-125 values were the only ones that contributed 
statistically significant to this model $(p=0.01)$, and the increase of this marker was associated with an increase in the likelihood of being diagnosed with infertility. We conducted that the elevated CA-125 values increased the risk of infertility.

The stage of endometriosis influences the fertility status. The statistical analysis of the second study suggests that the stage of endometriosis has a significant influence on fertility status $(X 2=23.19, p=0.00)$, with a magnitude of effect measured by Phi and Cramer's V very important. Practically, the advanced stages of endometriosis, even non-obstructive, can cause infertility through a mechanism other than the bilateral tubal obstruction.

After the statistical analysis, the IL- 6 was elevated in women with non-obstructive endometriosis and infertility $(p=0.00)$. The IL-8 did not show a statistical difference in non-obstructive endometriosis and there were no correlations between fertility and the IL- 8 levels.

The limitation of our studies was the number of patients and the fact that in the first study there was no significant difference in the stage of endometriosis over fertility, whereas in the second study our results show that there is an influence. This statistical difference is due to the different sample sizes.

These biomarkers of endometriosis are nonspecific inflammation associated proteins that reflect inflammatory changes also in other pathologies such as autoimmune disease, hepatitis, sepsis, pelvic inflammatory disease. Our findings could guide research to select a panel of cytokine with a better potential for diagnose in clinical practice. Furthermore, the association of CA-125, IL- 6 and IL-8 could improve diagnostic reliability.

Our data cannot be generalized, there is a need for a higher quality prospective studies and standardized approach, to draw firm conclusions regarding the role of biomarkers (CA-125, IL-6) in predicting infertility of women with non-obstructive endometriosis. This would provide more reliable and relevant information regarding this new hypothesis.

\section{Ethical approval and consent to participate}

This study was approved by the Institutional Review Board (IRB) of Filantropia Clinical Hospital. Written informed consent was obtained from all the participants and their participation was voluntary.

\section{Conflict of interest}

None.

\section{Funding}

This research did not receive any specific grant from funding agencies in the public, commercial, or not-for-profit sectors.

\section{REFERENCES}

1. Giudice L, Kao L. Endometriosis. The Lancet. 2004; 364(9447): 1789-1799, doi: 10.1016/s0140-6736(04)17403-5.

2. Fassbender A. Biomarkers of endometriosis. Fertility and Sterility. 2013; 99: 1135-1144.

3. Eskenazi B, Warner M. Epidemiology of endometriosis. Obstetrics and Gynecology Clinics of North America. 1997; 24(2): 235-258, doi: 10.1016/s0889-8545(05)70302-8.

4. Fassbender A, Burney RO, O DF, et al. Update on Biomarkers for the Detection of Endometriosis. Biomed Res Int. 2015; 2015: 130854, doi: 10.1155/2015/130854, indexed in Pubmed: 26240814.

5. Counseller V. Endometriosis. American Journal of Obstetrics and Gynecology. 1938; 36(5): 877-888, doi: 10.1016/s0002-9378(38)90579-4.

6. de Ziegler D, Pirtea P, Carbonnel M, et al. Implantation markers and endometriosis. Reprod Biomed Online. 2005; 11(4): 464-468, doi: 10.1016/s1472-6483(10)61142-x, indexed in Pubmed: 16274610.

7. Carmona F, Chapron C, Martínez-Zamora MÁ, et al. Ovarian endometrioma but not deep infiltrating endometriosis is associated with increased serum levels of interleukin-8 and interleukin-6. J Reprod Immunol. 2012; 95(1-2): 80-95, doi: 10.1016/j.jri.2012.06.001, indexed in Pubmed: 22819248.

8. Carp HJA, Selmi C, Shoenfeld Y. The autoimmune bases of infertility and pregnancy loss. J Autoimmun. 2012; 38(2-3): 266-274, doi: 10.1016/j. jaut.2011.11.016, indexed in Pubmed: 22284905.

9. May KE, Conduit-Hulbert SA, Villar J, et al. Peripheral biomarkers of endometriosis: a systematic review. Hum Reprod Update. 2010; 16(6): 1-24, doi: 10.1093/humupd/dmq009, indexed in Pubmed: 20462942.

10. Gupta S, Agarwal A, Sekhon L, et al. Serum and peritoneal abnormalities in endometriosis: potential use as diagnostic markers. Minerva Ginecol. 2006; 58(6): 527-551, indexed in Pubmed: 17108882.

11. Othman EED, Hornung D, Salem HT, et al. Serum cytokines as biomarkers for nonsurgical prediction of endometriosis. Eur J Obstet Gynecol Reprod Biol. 2008; 137(2): 240-246, doi: 10.1016/j.ejogrb.2007.05.001, indexed in Pubmed: 17582674.

12. Bedaiwy MA, Falcone T, Sharma RK, et al. Prediction of endometriosis with serum and peritoneal fluid markers: a prospective controlled trial. Hum Reprod. 2002; 17(2): 426-431, doi: 10.1093/humrep/17.2.426, indexed in Pubmed: 11821289

13. Ohata $\mathrm{Y}, \mathrm{Harada} \mathrm{T}$, Miyakoda $\mathrm{H}$, et al. Serum interleukin- 8 levels are elevated in patients with ovarian endometrioma. Fertil Steril. 2008; 90(4): 994-999, doi: 10.1016/j.fertnstert.2007.07.1355, indexed in Pubmed: 18635170

14. Salmeri FM, Laganà AS, Sofo V, et al. Behaviour of cytokine levels in serum and peritoneal fluid of women with endometriosis. Gynecol Obstet Invest. 2002; 54(2): 82-87, doi: 10.1159/000067717, indexed in Pubmed: 12566749 